\title{
Internet und Geschichte in Frankreich
}

\section{Mareike König et Annette Schläfer}

\section{(2) OpenEdition}

\section{Journals}

Édition électronique

URL : http://journals.openedition.org/ifha/7907

DOl : 10.4000/ifha.7907

ISSN : 2198-8943

\section{Éditeur}

IFRA - Institut franco-allemand (sciences historiques et sociales)

\section{Référence électronique}

Mareike König und Annette Schläfer, «Internet und Geschichte in Frankreich », Revue de I'IFHA [Online], HS | 2014, Online erschienen am: 01 September 2014, aufgerufen am 14 November 2019. URL : http:// journals.openedition.org/ifha/7907 ; DOI : 10.4000/ifha.7907

Ce document a été généré automatiquement le 14 novembre 2019.

(CIFHA 


\title{
Internet und Geschichte in Frankreich
}

\author{
Mareike König et Annette Schläfer
}

\section{Einleitung}

1 Wer über französische Geschichte oder mit französischen Quellen arbeitet, wird in Frankreich mit digitalen Angeboten reich versorgt. Die zentral aufgestellten Bibliotheks-, Dokumentations- und Forschungslandschaften erleichtern dabei zumeist Sucheinstiege und Zugang. Kennzeichnend für die französische Internetlandschaft sind groß angelegte, zentrale Digitalisierungsprojekte, zentrale Open-Access-Repositorien für Doktorarbeiten und andere wissenschaftliche Texte, eine ausgeprägte Fachkommunikation über Wissenschaftsblogs sowie ein zentraler Veranstaltungskalender und eine spezielle Suchmaschine für die Geistes- und Sozialwissenschaften. Dafür fehlen die Virtuellen Fachbibliotheken, wie man sie aus Deutschland kennt, ein Mailingservice wie H-Soz-u-Kult sowie kollaborative Nachweiswerkzeuge wie die Elektronische Zeitschriftenbibliothek (EZB) für E-Journals und das Datenbank-Infosystem (DBIS) für Datenbanken. Einige der Angebote zeichnen sich optisch durch eine größere Verspieltheit aus, als das deutsche Auge es von wissenschaftlichen Online-Angeboten diesseits des Rheins gewohnt ist ${ }^{1}$.

\section{Sucheinstieg für Internetressourcen, Datenbanken und digitalisierte Sammlungen}

2 Einen zentralen Sucheinstieg für im Open Access verfügbare Internetressourcen zu den Geisteswissenschaften in Frankreich bietet die Suchplattform Isidore ${ }^{2}$. Diese sehr leistungsstarke Suchmaschine wurde erst vor zwei Jahren speziell für die akademische Community entwickelt. Sie wertet Metadaten und Volltexte aus über 80 Sammlungen unterschiedlicher Herkunft aus, darunter die digitalisierten Zeitschriftenbestände von Persée, Revues.org und Cairn.info, die offenen Archive HAL und TEL, das zentrale 
Verzeichnis laufender französischer Dissertationen theses.fr, Digitalisate von Stadtbibliotheken, Sammlungen aus Gallica, Calenda, Hypotheses und viele weitere Angebote, die weiter unten vorgestellt werden. Die Suche in über 2,3 Millionen Dokumenten ist über Titel, Autor, Stichwort und Jahr möglich und kann im Nachhinein über verschiedene Facetten wie Fachdisziplin, Sammlung, Ressourcentyp, Sprache etc. eingeschränkt werden ${ }^{3}$.

3 Frankreich hat keinen eigenen zentralen Katalog für Datenbanken und OnlineRessourcen vergleichbar mit dem deutschen Datenbank-Infosystem (DBIS). Die französischen Bibliothekskataloge wie der Sudoc weisen zumeist nur kostenpflichtige Online-Angebote nach, und auch dies nur sehr unvollständig* ${ }^{4}$. Will man sich einen Überblick über Datenbanken im Bereich der Geschichtswissenschaften verschaffen, so bietet die kooperativ von mehreren Universitätsbibliotheken erstellte Website Signets des Universités ${ }^{5}$ einen Einstieg. Der Katalog erschließt seit April 2009 Internetseiten, Datenbanken, Kataloge, Blogs und E-Journals aus den Bereichen Geistes- und Sozialwissenschaften, Recht und Technik. Die Inhalte der Online-Angebote sind jeweils mit wenigen Sätzen beschrieben sowie anhand der Dewey-Dezimalklassifikation erschlossen. Die mobile Version der Signets bietet auch über Smartphones Zugang auf die derzeit rund 350 Online-Ressourcen im Bereich Geschichte und Geographie.

4 Eine Übersicht über digitalisierte Sammlungen von Dokumenten, Handschriften, Postkarten etc. aus französischen Bibliotheken und Archiven bietet der Katalog Catalogue des collections numérisées ${ }^{6}$ des Kultusministeriums. Komplementär dazu listete Numes $^{7}$ die Digitalisierungsprojekte der universitären Einrichtungen. Die Website wurde jedoch kürzlich gehackt und ist daher derzeit nicht zugänglich, mit unsicherem Ausgang. Die Übersicht Bases de données culturelles ${ }^{8}$ listet alphabetisch in Frankreich entstandene Datenbanken auf, die frei im Web verfügbar sind. Eine inhaltliche Erschließung über Metadaten fehlt allerdings. Ebenfalls nützlich ist die als Wiki aufgesetzte Übersicht zu digitalen Sammlungen Bibliopedia.

\section{a) Zeitschriften}

\section{Überblick über die Angebote}

5 Auch zur EZB gibt es in Frankreich, wie eingangs erwähnt, kein zentrales Pendant. Einen ähnlichen Aufbau wie der EZB findet man jedoch in den Online-RessourcenSammlungen vieler französischer Universitätsbibliotheken, die mit dem Programm AtoZ funktionieren. Von einem Äquivalent zur EZB kann man aber nicht sprechen, da die Listen vor allem die Abonnements der jeweiligen Bibliothek widerspiegeln. Es handelt sich also nicht um einen von mehreren Bibliotheken gemeinsam verwalteten Katalog. Nichtsdestotrotz bieten diese Online-Abonnement-Sammlungen zusätzlich zu den kostenpflichtigen und von der Universitätsbibliothek abonnierten OnlineZeitschriften zahlreiche im Internet frei zugängliche Journals und sind in jedem Fall für den ersten Überblick über eine Disziplin interessant. Man hat hier wie in der EZB die Möglichkeit, Listen von E-Journals nach Fächergruppen zu durchsuchen.

6 Es bietet sich daher für ein erstes Annähern an französische Zeitschriften und Datenbanken an, über die jeweilige französische Universitätsbibliothek in diesen Sammlungen die Fächergruppe »Histoire (Générale) et Histoire de l'Europe« zu durchforsten. 
7 In den Zeitschriftenauflistungen wird man zahlreiche weitere nützliche Hinweise auf Online-Plattformen und Möglichkeiten zum Online-Volltextzugriff auf die Zeitschriften finden. Die französische Infrastruktur für wissenschaftliche Zeitschriften ist extrem beeindruckend. Drei Online-Plattformen sind hierbei besonders hervorzuheben und in den einzelnen Rechercheschritten zu berücksichtigen. Die drei Plattformen haben jeweils ihr eigenes Profil und sind in ihrer Konzeption gut aufeinander abgestimmt:

Revues.org

8 Revues.org ${ }^{10}$ existiert seit 1999 und ist eine Editions- und Publikationsplattform für laufende Zeitschriften, die ausschließlich online oder in Papierform und elektronisch erscheinen und hierbei das Prinzip des (zumindest partiellen) Open Access respektieren. Dies bedeutet, dass eine Zeitschrift, die auf Revues.org präsent sein will, einen bestimmten Anteil ihrer bereits erschienen Nummern als kostenlosen Volltext anbieten muss.

Die Präsentationsseite von Revues.org bietet einen Katalog in einer alphabetischen Auflistung der derzeit über 400 Zeitschriften ${ }^{11}$. Da es sich ausschließlich um Zeitschriften der Geistes- und Sozialwissenschaften handelt, lohnt es sich, diese Liste zu sichten und für die einschlägigen Zeitschriften ein Monitoring über RSS-Feeds oder EMail-Alerts einzurichten.

Persée

10 Seit 2005 ist das Portal Persée ${ }^{12}$ online. Aufgabe von Persée ist es, im Gegensatz zu den laufenden Publikationen von Revues.org, Altbestände von Papierzeitschriften $\mathrm{zu}$ digitalisieren. Hierbei konzentriert sich Persée auf französischsprachige wissenschaftliche Zeitschriften (mit Redaktionskomitee) aus den Geistes- und Sozialwissenschaften, die von einem wissenschaftlichen Beirat ausgewählt und dann vom Projekt Persée digitalisiert werden. Der Server enthält mittlerweile weit über 300.000 Dokumente.

11 Eine sehr praktisch gestaltete Benutzeroberfläche bietet neben dem Zugang zum Volltext der Zeitschriften einige weitere nützliche Funktionalitäten. Man kann zum Beispiel die Zeitschriftenliste nach vertretenen Fachrichtungen durchblättern und dabei für die Geschichtswissenschaften auf wichtige Referenzzeitschriften wie die "Annales«. "Histoire«, "Sciences sociales«, die »Annales historiques de la Révolution française«, »Médiévales« oder "Vingtième Siècle« stoßen. Persée bietet des Weiteren strukturierte und angereicherte Metadaten, nach dem internationalen Standard TEI organisierte Texte und eine garantierte Langzeitarchivierung über Digital object Identifier (DOI).

Cairn.info

12 Cairn.info ${ }^{13}$ ist ein kommerzielles Portal, das von vier französischen und belgischen Verlagen (Belin, De Boeck, La Découverte und Erès) aufgesetzt worden ist, um gemeinsam ihre geistes- und sozialwissenschaftlichen Zeitschriften zu vertreiben. Auch hier kann man die ausgewerteten Zeitschriften nach Fächergruppen durchblättern und einschlägige Publikationen für die Geschichtswissenschaft finden.

Die drei genannten Portale sind nicht nur konzeptionell gut aufeinander abgestimmt, sondern dank automatischem Datenaustausch auch technisch miteinander vernetzt. Nutzerinnen und Nutzer einer Zeitschrift wie z.B. "Médiévales ${ }^{14}$ können somit problemlos auf dem Portal Revues.org auf laufende Open Access-Texte zugreifen, 
werden für kostenpflichtige aktuelle Nummern auf Cairn.info weitergeleitet und für digitalisierte Papierjahrgänge auf Persée.

Zusätzlich zu den drei genannten wichtigsten Zeitschriftenplattformen sollen hier noch kurz einige weitere vorgestellt werden.

BiblioSHS

BiblioSHS ${ }^{15}$ ist eine Informationsplattform, die leider nur für französische Forschende zugänglich ist, die dem Centre national de la recherche scientifique (CNRS) angegliedert sind. Die Plattform dient zur Administration der elektronischen Ressourcen, für die das CNRS eine globale Lizenz erworben hat. Somit können Wissenschaftlerinnen und Wissenschaftler, die im Besitz eines CNRS-Logins sind, von jedem beliebigen Arbeitsplatz auf die abonnierten Online-Journals und Datenbanken zugreifen.

Das Zeitschriftenverzeichnis von BiblioSHS kann allerdings auch ohne vorheriges Login nach Disziplinen durchsucht werden und umschließt dabei wohl am umfassendsten die Angebote der französischen und englischsprachigen historischen Fachzeitschriften, denn es bündelt die Informationen der Plattformen:

- OpenEdition (incl. revues.org)

- Persée

- Cairn

- Erudit

- JSTOR

- Project MUSE

- ScienceDirect

- SpringerLink

Erudit

17 Erudit $^{16}$ ist eine kanadische, dem Open Access verpflichtete Plattform für wissenschaftliche Fachzeitschriften, die 1998 von den Presses de l'Université de Montréal ins Leben gerufen wurde. Heute wird sie gemeinschaftlich von der Université de Montréal, der Université Laval und der Université du Québec in Montréal betreut und aktualisiert. Neben Zeitschriften findet man auf Erudit auch Doktorarbeiten, elektronische Bücher, Kolloquiumsveröffentlichungen und andere Texte und Dokumente im Open Archive Server. Auch Erudit bietet die auf der Plattform vertretenen Zeitschriften (und die der Partnerplattform Persée) in einer Liste nach Fächergruppen sortiert zum Durchsuchen und für eine erste Orientierung an.

Weitere Projekte für die Bereitstellung digitalisierter Volltexte sind in Planung oder in Vorbereitung, wie beispielsweise Biblissima ${ }^{17}$, eine Meta-Bibliothek von bereits digitalisierten Beständen westeuropäischer Handschriften vom Mittelalter bis zur Renaissance.

Weitere internationale Anbieter: Project Muse und JSTOR

19 Französischsprachige Zeitschriften bzw. Zeitschriften zur französischen Geschichte gibt es neben den französischen auch bei internationalen Anbietern wie Project Muse und JSTOR. Beide sind amerikanische Retrodigitalisierungsplattformen mit geistes- und sozialwissenschaftlicher Ausrichtung und dürften den deutschen Wissenschaftlerinnen und Wissenschaftlern bekannt sein. In Project Muse findet man zum Thema französische Geschichte vor allem englischsprachige Fachzeitschriften (z. B. »French Historical Studies«), Zeitschriften zur Geschichte Afrikas oder zur Kolonialgeschichte 
und französischsprachige kanadische Zeitschriften (z.B. »Histoire sociale/Social history«). JSTOR enthält auch einige französische Fachzeitschriften (z. B. »Guerres mondiales et conflits contemporains «, „L'Actualité de l'histoire, Bulletin annuel de l'Institut français d'histoire sociale«, »Revue d'histoire moderne et contemporaine«, »Revue historique« und andere) sowie englischsprachige Zeitschriften zur europäischen Geschichte. Die Angebote müssen (wie in Deutschland) von den Bibliotheken abonniert sein, damit man über eine Campuslizenz darauf zugreifen kann. Für JSTOR ist dies in einem Großteil der französischen Universitäts- und Forschungsbibliotheken der Fall.

\section{Einen bestimmten Artikel finden und auf das Dokument zugreifen}

Beim Erstellen einer Bibliographie, sei es für eine Hausarbeit, einen Artikel oder für die Doktorarbeit, wird man das sogenannte "Schneeballsystem" nutzen, indem das Literaturverzeichnis von bereits gefundenen wichtigen Veröffentlichungen zum entsprechenden Thema nach weiteren bibliographischen Hinweisen durchgegangen wird. In diesem Fall hat man die vollständige bibliographische Beschreibung der Dokumente und muss nun Wege finden, um auf den Text in elektronischer Form oder in Papierform zugreifen zu können.

22 Erste Anlaufstelle kann hierfür Mir@bel (Mutualisation d'informations sur les revues et leurs accès dans les bases en ligne $)^{18}$ sein, denn selbst wenn man im Laufe der Zeit immer wieder auf die gleichen Fachzeitschriften zurückgreift und Erfahrungswissen ansammelt, wo und wie man am schnellsten an den Volltext einer Veröffentlichung kommt, so weiß man doch nicht immer, ob eine Zeitschrift in elektronischer oder gedruckter Form vorliegt. Das Projekt Mir@bel, das kollektiv von französischen Universitätsbibliotheken und Forschungseinrichtungen geführt wird, sammelt Informationen zum Zugang zu Metadaten (Inhaltsverzeichnisse, Abstracts) und Volltexten für wissenschaftliche Zeitschriften.

23 Über Datenimport werden die Informationen $\mathrm{zu}$ den Zeitschriften der Portale Revues.org, Persée, Cairn.info und Erudit.org immer aktuell in Mir@bel widergespiegelt, was das Tool zu einem unumgänglichen Rechercheinstrument macht. Es ist jedoch zu beachten, dass man in Mir@bal immer nur nach Zeitschriftentiteln suchen kann. Einzelne Artikel sind hier nicht verzeichnet.

\section{b) Volltexte}

\section{Retrodigitalisierte Quellen}

Gallica

Gallica $^{19}$ ist die französische nationale digitale Bibliothek. Betrieben wird dieses Portal von der französischen Nationalbibliothek (Bibliothèque nationale de France, BNF), deren digitalisierten Bestände sie ursprünglich präsentieren sollte. Inzwischen sind der digitalen Bibliothek Gallica weitere Partnerinstitutionen angegliedert, deren Digitalisate durch automatischen Datenaustausch (OAI-PMH) ebenfalls in der Portalnavigation und in der Suchmaske von Gallica angezeigt werden. Somit können die wichtigsten französischen digitalen Sammlungen über das Gallica-Portal durchsucht werden. 
Als französische Antwort auf das weltweite Bücher-Digitalisierungsprojekt von Google konzipiert, werden für Gallica vor allem französischsprachige Dokumente eingescannt. Es finden sich aber auch italienische, englische, lateinische griechische, spanische und fast 10.000 deutschsprachige Werke in der digitalen Sammlung. Der Schwerpunkt der Digitalisierung von Gallica lag zunächst auf schwer auffindbaren, raren oder vergriffenen Werken von wissenschaftlichem Interesse. Nach und nach entwickelt sich Gallica nun zu einer multimedialen Plattform und bietet neben Büchern auch digitalisierte Zeitschriften, Bilder, Karten, Tondateien und Partituren. Der Ausbau der Bild- und Tonbestände wird in den Projektleitlinien als prioritär angegeben, gleich nach der Digitalisierung der Zeitschriften- und Pressebestände vor allem des 19. Jahrhunderts.

Gallica ist Partner der europäischen digitalen Bibliothek Europeana ${ }^{20}$ und liefert Digitalisate und Metadaten an das europäische Großprojekt.

Handschriften: Kataloge und Digitalisate

Mittelalterliche und frühneuzeitliche Handschriften sind in Frankreich in allen Bibliothekstypen vorhanden. Um den Zugang zu zentralisieren, gibt es verschiedene gemeinschaftliche Handschriftenkataloge und virtuelle Bibliotheken, die sich gegenseitig ergänzen. Dennoch muss man auf der Suche nach Handschriften mehrere Kataloge verwenden: Der Verbundkatalog Calames ${ }^{21}$ verzeichnet Archivalien und Handschriften, die in den französischen Universitätsbibliotheken aufbewahrt werden. Für die europäischen und orientalischen Handschriften, die sich in der BnF befinden, gibt es jeweils eigene Kataloge, die online zur Verfügung stehen ${ }^{22}$. Handschriften aus den öffentlichen Bibliotheken sind dagegen im Catalogue Collectif de France ${ }^{23}$ erfasst.

Beschreibungen und Digitalisate der Handschriften der BnF kann man über die Bilddatenbank Mandragore ${ }^{24}$ recherchieren. Den wertvollen Altbestand der Stadtbibliotheken stellen diese zumeist über ihre eigenen Websites zur Verfügung ${ }^{25}$. In der 2013 an den Start gegangenen Bibliothèque virtuelle des manuscrits médiévaux $(B V M M)^{26}$ des Institut de recherche et d'histoire des textes (IRHT) ${ }^{27}$ werden digitalisierte Manuskripte, die nicht im Besitz der BnF sind, zusammengeführt ${ }^{28}$. Auch die Staatsbibliothek zu Berlin liefert einige hundert Digitalisate ihrer Handschriften an diese digitale Bibliothek.

Bei der Suche nach digitalisierten mittelalterlichen Manuskripten kann darüber hinaus die Website Ménéstrel (siehe unten) nützlich sein, wo einige Artikel auch auf Deutsch publiziert werden. Darüber hinaus gibt es weitere sehr nützliche Übersichten zu digitalisierten Handschriften, wie beispielsweise die Unterseiten Bibliothèques numériques $^{29}$ auf dem bereits erwähnten Wiki Bibliopédia ${ }^{30}$ oder, über den französischsprachigen Raum hinaus, bei Archivalia ${ }^{31}$.

\section{Aktuelle digitale Forschungsliteratur}

Open Archive Server

30 Mit dem multidisziplinären Open Archive Server HAL $^{32}$ (hyper articles en ligne) steht der französischen Wissenschaftscommunity ein zentrales Repositorium zur Selbstarchivierung wissenschaftlicher Texte zur Verfügung. Für die Geisteswissenschaften gibt es ein eigenes Unterarchiv (bzw. eine eigene Benutzeroberfläche): HAL-SHS ${ }^{33}$ für »Sciences de l'Homme et de la Société«. Der weitaus größte Anteil an selbstarchivierten Texten in HAL-SHS sind laut einer Analyse vom 7. 
Mai 2013 Artikel aus Zeitschriften mit wissenschaftlichem Beirat, gefolgt von Konferenzbeiträgen, Preprints und Veröffentlichungen einzelner Kapitel. Die Geschichtswissenschaften sind derzeit mit über 5.000 Artikeln vertreten. in Planung. Vorgesehen ist dabei, die Idee der Nationallizenz mit einer zentralen Informations- und Rechercheplattform zu verbinden und so Fachzeitschriften, E-Books und andere wissenschaftliche Dokumente zugänglich zu machen. Durchgeführt wird das Projekt, dessen Abschluss für das Jahr 2014 vorgesehen ist, von vier Institutionen: 
dem Centre National de la Recherche Scientifique (CNRS), der Agence Bibliographique de l'Enseignement Supérieur (ABES), dem Consortium Universitaire de Publications Numériques (Couperin) und der Université de Lorraine (für die Conférence des Présidents d'Université - CPU).

\section{c) Thematische Websites}

Das Angebot an französischen thematischen Websites, die für historisch Forschende interessant sind, ist natürlich riesig groß. Aufgrund der hier gebotenen Kürze wird lediglich auf die zentrale Einstiegsseite der einzelnen Epochen verwiesen. Dort finden sich jeweils weiterführende Links. Die bereits erwähnten Sucheinstiege für Internetressourcen wie Signets oder Numes können natürlich ebenso für das Aufspüren thematischer Websites verwendet werden.

Ein zentraler Einstieg für Mediävisten ist das Portal Ménéstrel ${ }^{41}$, das neben einer kritisch kommentierten Linksammlung sowie einer Zusammenstellung von Bibliographien, Handschriftenkatalogen, Texten zu Computer und Mediävistik Hinweise zum Studium der Mediävistik in verschiedenen Ländern enthält sowie Bibliotheken und Rechercheinstrumente vorstellt. Für Mediävisten zentral ist darüber hinaus die Website der École nationale de Chartes ${ }^{42}$, die auch Online-PaläographieKurse anbietet, wie man sie ebenfalls auf den Seiten von französischen Archiven findet $^{43}$. $\mathrm{Zu}$ nennen ist hier schließlich die Website des Institut de recherche et d'histoire des textes (IRHT), das der Erforschung mittelalterlicher Handschriften und alter Drucke verpflichtet ist ${ }^{44}$.

Für die Epoche der Frühen Neuzeit gibt es keine zentrale Einstiegsseite in Frankreich. Man findet jedoch die Websites einzelner Forschungszentren wie z. B. die Société Française d'Étude du Dix-Huitième Siècle ${ }^{45}$, die auf Neuerscheinungen, Kolloquien und Dissertationen verweist sowie die Bibliographie zum 18. Jahrhundert ab dem Berichtsjahr 2001 online bereitstellt. Darüber hinaus gibt es zahlreiche Seiten zu Napoleon oder zur Französischen Revolution (z. B. Fondation Napoléon ${ }^{46}$ mit ihrer digitalen Bibliothek zu Napoléon ${ }^{47}$ oder das Institut d'histoire de la Révolution française $)^{48}$. Die Bibliothèques Virtuelles Humanistes ${ }^{49}$ existiert seit 2002 und sammelt digitalisierte Quellen, Texte und notarielle Akten aus der Zeit des Humanismus und der Renaissance überwiegend aus der französischen Region Centre.

Als Einstieg für das 19. Jahrhundert kann die Website des Centre de recherche XIX ${ }^{\mathrm{e}}$ siècle $^{50}$ der Sorbonne dienen, die Informationen zu Lehrangeboten, Kolloquien und Forschungsvorhaben des Lehrstuhls enthält. Zentral ist die Zeitschrift Revue d'histoire du XIX ${ }^{\mathrm{e}}$ siècle ${ }^{51}$ mit einem Schwerpunkt auf der Revolutions- und Sozialgeschichte. Die Website bietet Zugriff auf die freigeschalteten Volltexte sowie Informationen zu aktuellen Tagungen und Dissertationsvorhaben. Ergänzt wird das Informationsangebot durch ein Blog ${ }^{52}$ bei hypotheses.org.

41 Für Zeithistoriker ist das Webangebot des Institut d'histoire du temps présent ${ }^{53}$ von Interesse. An dieser namhaften Pariser Forschungseinrichtung wird über Weltgeschichte ab 1914 geforscht. Auf der Website werden die Forschungsprojekte des Instituts vorgestellt. In der Rubrik »Ressources en ligne« werden Online-Dokumente angeboten, so z. B. das Bulletin des Instituts, Texte, Bibliographien und die Volltextdatenbank »Frankreich im Zweiten Weltkrieg«, die in Zusammenarbeit mit dem DHI Paris veröffentlicht wurde. Sie enthält die Edition der Lageberichte des 
Militärbefehlshabers Frankreichs und der Synthesen der Berichte der französischen Präfekten der Jahre 1940-194454. Wer sich für europäische Integrationsgeschichte interessiert, kann das Portal der internationalen Forschergruppe RICHIE als zentralen Einstieg nutzen ${ }^{55}$.

Zum 100. Jahrestag des Ersten Weltkriegs, der in Frankreich sehr viel stärker präsent ist als in Deutschland, gibt es zahlreiche Online-Projekte und Online-Publikationen. Zentraler Einstieg ist das speziell dafür aufgesetzte Portal der Mission du Centenaire ${ }^{56}$, auf dem zahlreiche digitalisierte Quellen präsentiert werden.

\section{d) Online-Bibliographien}

Die Bibliographie de l'histoire de France (BHF) gibt es bedauerlicherweise nur als gedruckte Jahresberichte, die folglich mit einiger Verzögerung zum Berichtsjahr erscheinen. Ein geplantes Projekt, die seit 1959 existierende BHF für die Jahre 2004-2007 retrodigitalisiert online zu stellen, wartet immer noch seiner Umsetzung. Rückfragen bei den Projektverantwortlichen ergaben, dass mit einer nahen Veröffentlichung nicht zu rechnen ist, obwohl die Website eine andere Auskunft gibt ${ }^{57}$.

Online zugänglich ist dagegen die französische Nationalbibliographie ${ }^{58}$. Aufgrund ihres Umfangs ist sie jedoch in der Benutzung etwas sperrig. Auch enthält sie keine unselbstständigen Werke wie z. B. Aufsätze. Es werden nur die Dokumententypen Bücher, Zeitschriften, Musik, Karten und audiovisuelle Medien unterschieden. Die Neulieferungen für Bücher erscheinen vierzehntägig und werden nach der DeweyDezimalklassifikation thematisch geordnet.

\section{e) Fachkommunikation}

45 Veranstaltungen und Calls for papers aus dem Bereich der Geistes- und Sozialwissenschaften werden in Frankreich bei Calenda ${ }^{59}$ angekündigt, das mit zum Portal OpenEdition gehört. Es ist in dieser Hinsicht das Pendant zur deutschen Mailingliste H-Soz-u-Kult, nur dass es alle Disziplinen der Geistes- und Sozialwissenschaften betrifft. Die Annoncen werden auch nicht per Mail verschickt, sondern man kann die neuen Ankündigungen für die eigenen Interessensbereiche per RSS-Feed abonnieren. Dabei ist ein sehr viel feineres Filtern möglich. Frankreich selbst hat keinen direkten Ableger der H-Net-Familie. Rezensionen, Calls for papers, Konferenzen, Jobangebote etc. werden jedoch auch über die englischsprachige elektronische Diskussionsliste H-France ${ }^{60}$ abgedeckt.

Fachkommunikation wird in Frankreich auch über Wissenschaftsblogs betrieben, die im Bereich der Geistes- und Sozialwissenschaften mit dem Portal fr.hypotheses.org ${ }^{61}$ einen zentralen Einstieg haben. Die Wissenschaftsblogs heißen hier »carnets de recherche", was nicht unwesentlich $\mathrm{zu}$ ihrem Erfolg und damit zum Vorsprung Frankreichs in Sachen Wissenschaftsbloggen beigetragen haben dürfte. Doch die deutschsprachigen Länder holen auf: Hypotheses.org hat seit März 2012 mit de.hypotheses.org ${ }^{62}$ auch eine deutsche Einstiegsseite. Es lohnt sich, den Katalog der Wissenschaftsblogs durchzusehen, der einen gezielten Zugriff erlaubt. Die Blogs sind dort sowohl thematisch als auch chronologisch geordnet ${ }^{63}$. 


\section{f) Bilddatenbanken}

Das Portal Joconde ${ }^{64}$ des französischen Kulturministeriums enthält die Kunstbestände der französischen Nationalmuseen und ist damit die wichtigste Bilddatenbank in Frankreich. Daneben bietet der Louvre in mehreren Teilkatalogen z. B. zu graphischen Werken oder zu Keramiken seine eigenen Bilddatenbanken $a^{65}$. Weitere Bildnachweise können in den bereits erwähnten Catalogue des collections numérisées ${ }^{66}$ oder im Wiki Bibliopedia ${ }^{67}$ ermittelt werden. Eine französische Besonderheit ist die Seite MédiHAL ${ }^{68}$, offenes Archiv und Sharing-Plattform speziell für wissenschaftliche Fotos und Abbildungen. Es handelt sich dabei um das Pendant des oben erwähnten Repositoriums für wissenschaftliche Texte HAL-SHS.

\section{Zum Schluss}

Das Internet ist ein schnelllebiges Medium, und obwohl die hier vorgestellten Webangebote alle langfristig angelegt sind, werden erfahrungsgemäß in Kürze einige der zitierten Links ins Leere führen, während weitere zentrale Angebote hinzugekommen sind. Mit unserem Gemeinschaftsblog Franco-Fil ${ }^{69}$ bieten wir eine Möglichkeit, bezüglich der wissenschaftlichen Online-Angebote auf dem Laufenden zu bleiben. Darin werden Hinweise und Tipps für die Informationssuche gegeben sowie neue Online-Ressourcen für die geisteswissenschaftliche Forschung zu Frankreich vorgestellt. Thematische Anregungen sowie Gastbeiträge für das Blog nehmen wir gerne entgegen!

\section{NOTES}

1. Für einen Gesamtüberblick zu digitalen Angeboten in Frankreich siehe auch Mareike König, Guide Frankreich, in: Clio-online, 27.04.2012, http://www.clio-online.de/guides/frankreich/ koenig2012. Alle Links wurden am 15.07.2014 überprüft.

2. Isidore, http://www.rechercheisidore.fr/.

3. Für weitere Erläuterungen zur Verwendung von Isidore siehe Kaja Antonowicz, Mittwochstipp

7: ISIDORE - Meta-Suchmaschine der französischen Geisteswissenschaften, in: Francofil, 28.08.2013, http://francofil.hypotheses.org/826.

4. $\mathrm{Zu}$ den Bibliotheken und Bibliothekskatalogen in Frankreich siehe den Beitrag von Dominique Bouchery und Mareike König »Bibliotheken in Frankreich: Einrichtungen, Bestände und Suchstrategien« in diesem Wegweiser (http://ifha.revues.org/7889).

5. Signets des Universités, http://www.signets-universites.fr/.

6. Catalogue des collections numérisées, http://www.numerique.culture.fr/pub-fr/index.html.

7. Numes, http://www.numes.fr/.

8. Bases de données culturelles, http://www.culturecommunication.gouv.fr/Ressources/Basesde-donnees.

9. Bibliopedia, http://www.bibliopedia.fr/index.php/Biblioth\%C3\%A8ques_num\%C3\%A9riques.

10. Revues.org, http://www.revues.org/. 
11. Revues.org, Katalog, http://www.openedition.org/catalogue-journals.

12. Persée, http://www.persee.fr.

13. Cairn.info, http://www.cairn.info/.

14. Médiévales, http://medievales.revues.org.

15. BiblioSHS, http://biblioshs.inist.fr/.

16. Erudit, http://www.erudit.org/.

17. Biblissima, http://www.biblissima-condorcet.fr/.

18. Mir@bel, http://www.reseau-mirabel.info/.

19. Gallica, http://gallica.bnf.fr/.

20. Europeana, http://www.europeana.eu/.

21. Calames, http://www.calames.abes.fr/.

22. BnF, http://www.bnf.fr/fr/collections_et_services/acces_aux_catalogues_numerises.html.

23. Catalogue collectif de France, http://ccfr.bnf.fr/portailccfr/jsp/index.jsp.

24. Mandragore, http://mandragore.bnf.fr/html/accueil.html.

25. Siehe dazu den Beitrag von Dominique Bouchery und Mareike König »Bibliotheken in Frankreich: Einrichtungen, Bestände und Suchstrategien« in diesem Wegweiser (http:// ifha.revues.org/7889).

26. Bibliothèque virtuelle des manuscrits médiévaux, http://bvmm.irht.cnrs.fr/.

27. Institut de recherche et d'histoire des textes, http://www.irht.cnrs.fr.

28. Zur Kritik der Website siehe Klaus Graf, BVMM - Bibliothèque virtuelle des manuscrits médiévaux, in: Archivalia, 2.04.2013, http://archiv.twoday.net/stories/326529091/.

29. Bibliothèques numériques, http://www.bibliopedia.fr/index.php/ Biblioth\%C3\%A8ques_num\%C3\%A9riques.

30. Bibliopedia, http://www.bibliopedia.fr/index.php/Biblioth\%C3\%A8ques_num\%C3\%A9riques.

31. Klaus Graf, Lists of digitized manuscript catalogs and multi-library medieval manuscript databases, in: Archivalia, 8.08.2013, http://archiv.twoday.net/stories/453138863/.

32. HAL, http://hal.archives-ouvertes.fr/.

33. HAL-SHS, http://halshs.archives-ouvertes.fr/.

34. TEL, http://tel.archives-ouvertes.fr/.

35. Open Edition Books, http://books.openedition.org.

36. Zum Freemium-Modell von OpenEdition siehe Mareike König, Neue Plattform OpenEdition Books, in: Digital Humanities am DHIP, 21.02.2013, http://dhdhi.hypotheses.org/1553.

37. Nationallizenzen, http://www.nationallizenzen.de/.

38. Licences nationales, http://www.licencesnationales.fr.

39. Couperin, http://www.couperin.org/.

40. Istex, http://www.istex.fr/.

41. Ménéstrel, http://www.menestrel.fr/.

42. École nationale de Chartes, http://www.enc.sorbonne.fr/.

43. Vgl. Sven Ködel, Mittwochstipp 5: Paläographische Online-Kurse in Frankreich, in: Franco-Fil, 9.05.2013, http://francofil.hypotheses.org/742.

44. Institut de recherche et d'histoire des textes, http://www.irht.cnrs.fr/.

45. Société Française d'Étude du Dix-Huitième Siècle, http://sfeds.ish-lyon.cnrs.fr/.

46. Fondation Napoléon, http://www.napoleon.org/fr/fondation/.

47. La Bibliothèque numérique napoléonienne, http://www.napoleon.org/fr/napoleonica/ bibliotheque/index.asp.

48. Institut d'histoire de la Révolution française, http://ihrf.univ-paris1.fr/.

49. Les Bibliothèques Virtuelles Humanistes, http://www.bvh.univ-tours.fr/.

50. Centre de recherche XIXe siècle, http://www.univ-paris1.fr/centres-de-recherche/crhxix/.

51. Revue d'histoire du XIX ${ }^{\mathrm{e}}$ siècle, http://rh19.revues.org/.

52. Actualité du XIX ${ }^{\mathrm{e}}$ siècle, http://histoire19.hypotheses.org/. 
53. Institut d'histoire du temps présent, http://www.ihtp.cnrs.fr/.

54. Frankreich im Zweiten Weltkrieg, http://www.ihtp.cnrs.fr/prefets/.

55. RICHIE, http://www.europe-richie.org.

56. Mission Centenaire, http://centenaire.org/fr.

57. Vgl. die Projektwebsite, http://bhf.revues.org/index.html.

58. Bibliographie nationale française, http://bibliographienationale.bnf.fr/.

59. Calenda, http://calenda.org/.

60. H-France, http://www.h-france.net/.

61. Carnets de recherche fr.hypotheses.org, http://fr.hypotheses.org/.

62. Deutschsprachiges Blogportal für die Geistes- und Sozialwissenschaften, http:// de.hypotheses.org.

63. Zu den Blogs siehe auch Mareike König, Wissenschaftliche Blogs zwischen Deutschland und Frankreich: die Blogplattform Hypotheses.org, in: Michel Grunewald (Hg.), France-Allemagne au $\mathrm{XX}^{\mathrm{e}}$ siècle. La production de savoir sur l'autre. IV Les médias, Bern 2014 (im Druck). Zu deutschsprachigen Geschichtsblogs siehe: Klaus Graf, Mareike König, Forschungsnotizbücher im Netz: Weblogs in der deutschsprachigen Geschichtswissenschaft, in: WerkstattGeschichte 61 (2012), S. 76-87, PDF: http://werkstattgeschichte.de/werkstatt_site/archiv/ WG61_076-087_GRAF_NETZ.pdf.

64. Joconde, http://www.culture.gouv.fr/documentation/joconde/fr/pres.htm.

65. Le Louvre, http://www.louvre.fr/moteur-de-recherche-oeuvres.

66. Catalogue des collections numérisées, http://www.numerique.culture.fr/pub-fr/index.html.

67. Bibliopedia, http://www.bibliopedia.fr/index.php/Biblioth\%C3\%A8ques_num\%C3\%A9riques.

68. MédiHAL, http://medihal.archives-ouvertes.fr/.

69. Franco-Fil, http://francofil.hypotheses.org/.

\section{RÉSUMÉS}

Frankreich verfügt in puncto Online-Edition in den Geistes- und Sozialwissenschaften über eine herausragende Infrastruktur. Elektronische Zeitschriftenportale, Open Access-Repositorien, Wissenschaftsblogs und eine wissenschaftliche Kommunikationsplattform sind zentral organisiert und erleichtern somit den ersten Einstieg in die französische Recherchelandschaft. In diesem Artikel werden zentrale Sucheinstiege für Datenbanken, E-Journals, Volltexte, Handschriften und Bilddatenbanken vorgestellt. Ergänzend werden einige für Historikerinnen und Historiker bedeutende thematische Websites präsentiert.

\section{INDEX}

Schlüsselwörter : Frankreich, Geschichtswissenschaft, Geschichte, Internet, Bibliographie, Datenbank, Online-Publikation, Open Access 


\section{AUTEURS}

\section{MAREIKE KÖNIG}

Dr. Mareike König ist Leiterin der Bibliothek und der Abteilung 19. Jahrhundert am Deutschen Historischen Institut Paris sowie Leiterin der Redaktion des deutschsprachigen Blogportals für die Geisteswissenschaften de.hypotheses.org.

Mail: mkoenig[at]dhi-paris.fr

\section{ANNETTE SCHLÄFER}

Annette Schläfer ist Wissenschaftliche Dokumentarin und Webmaster am Centre interdisciplinaire d'études et de recherches sur l'Allemagne (CIERA) in Paris.

Mail: Schlafer[at]ciera.fr 